\title{
FATIGUE ANALYSIS OF A REACTOR PRESSURE VESSEL FOR SMART
}

\author{
MYUNG JO JHUNG \\ Research Management Department, Korea Institute of Nuclear Safety \\ 62 Gwahak-ro, Yuseong-gu, Daejeon, 305-338 Korea \\ E-mail : mjj@kins.re.kr \\ Received June 13, 2011 \\ Accepted for Publication July 16, 2011
}

The structural integrity of mechanical components during several transients should be assured in the design stage. This requires a fatigue analysis including thermal and stress analyses. As an example, this study performs a fatigue analysis of the reactor pressure vessel of SMART during arbitrary transients. Using heat transfer coefficients determined based on the operating environments, a transient thermal analysis is performed and the results are applied to a finite element model along with the pressure to calculate the stresses. The total stress intensity range and cumulative fatigue usage factor are investigated to determine the adequacy of the design.

KEYWORDS : Reactor Pressure Vessel, Heat Transfer Coefficient, Fatigue, Stress Concentration Factor, Fatigue Curve, Rain-flow Method, Cumulative usage Factor

\section{INTRODUCTION}

SMART (System-Integrated Modular Advanced Reactor) is a first-of-its-kind integral reactor capable of generating $330 \mathrm{MW}$ of power for power generation and seawater desalination. SMART employs various design features that are not typically found in other nuclear power plants. Examples include a unique passive residual heat removal system, an enclosure for a pressurizer, eight helical steam generators, and four canned reactor coolant pumps inside a reactor pressure vessel. The integrated arrangement of these components enables the removal of the large pipe connections between the major reactor coolant systems, therefore fundamentally eliminating the possibility of a large-break loss-of-coolant accident [1].

There are many transients considered in the design stage. Their effect on the structural integrity should be addressed in the licensing documents in the form of a design report. Therefore in this study, an analysis procedure for fatigue analysis is suggested that includes thermal and stress analyses.

For the transient thermal analysis, the heat transfer coefficients are determined based on the operating environment and the thermal transient data are simplified to prepare a straightforward input deck. The most severe instances are found considering the total stress intensity range and the stress levels at those times are obtained along with the applied pressure. These values are then used in a fatigue analysis to determine the final cumulative usage factor.

Example analyses are performed for the reactor pressure vessel of SMART for arbitrary transients, and two acceptance criteria, the primary plus the secondary stress intensity and cumulative usage factor, are investigated.

\section{ANALYSIS}

\subsection{Finite Element Models}

The reactor pressure vessel of SMART is considered in this study. It is made of ASME SA-508 Grade 3 Class 1 material [1]. Flanges are not included in the model in this study, which should be investigated separately in detail.

Two finite element models are developed for a transient thermal analysis and a structural analysis using ANSYS [2]. For the thermal analysis, 2-D thermal solid elements are used in the reactor vessel and for the cladding. This element can be used as an axisymmetric ring element with 2-D thermal conduction capability. It has four nodes with a single degree of freedom, the temperature, at each node. Six elements exist in the radial direction of the shell to represent the profile of the result in a manner suitable for generating sufficient information in the ensuing analysis.

For the structural analysis, 2-D structural solid elements are used, as shown in Figure 1. These types of elements are used for the 2-D modeling of solid structures. They 


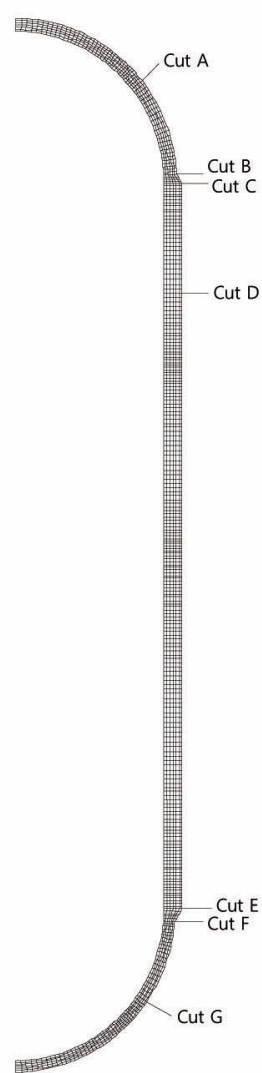

Fig. 1. Finite Element Model and Locations for Calculating the Fatigue usage Factor

can be used as axisymmetric elements. An element in this case is defined as having four nodes with two degrees of freedom for each node; these are the translations in the nodal $\mathrm{x}$ and $\mathrm{y}$ directions. Symmetric boundary conditions are imposed at the center nodes of the upper and lower heads. In addition, one node is fixed in all directions so as not to generate rigid body motion.

\subsection{Transients}

Several transients are considered in this study, as summarized in Table 1 . These are arbitrarily chosen for the fatigue analysis. The typical pressure and temperature histories for the plant heat-up and cool-down processes are respectively shown in Figures 2 and 3.

\subsection{Thermal Analysis}

To obtain the temperature distribution in the shell and head of the vessel, transient thermal analyses are performed for each transient defined previously. 2-D thermal surface effect elements for the thermal analysis are used for various surface effect applications, such as the heat transfer coefficient, which may be overlaid onto a face of any 2-D thermal solid element.
Table 1. Transient Summaries

\begin{tabular}{c|c|l|c}
\hline Condition & ID & \multicolumn{1}{c|}{ Description } & Occurrences \\
\hline \multirow{2}{*}{$\begin{array}{c}\text { Normal } \\
\text { operation } \\
\text { (Level A) }\end{array}$} & N08 & Plant heat-up & 300 \\
\cline { 2 - 4 } & N9A & Plant cool-down & 300 \\
\cline { 2 - 4 } & N9B & Pressurizer flooding & 1 \\
\hline \multirow{2}{*}{ Test } & $\begin{array}{l}\text { RCS hydraulic and } \\
\text { leak test in the } \\
\text { increasing direction }\end{array}$ & 15 \\
\cline { 2 - 4 } & T1B & $\begin{array}{l}\text { RCS hydraulic and } \\
\text { leak test in the } \\
\text { decreasing direction }\end{array}$ & 15 \\
\hline $\begin{array}{c}\text { Upset } \\
\text { (Level B) }\end{array}$ & U06 & $\begin{array}{l}\text { Decrease in RCS } \\
\text { inventory }\end{array}$ & 30 \\
\hline
\end{tabular}

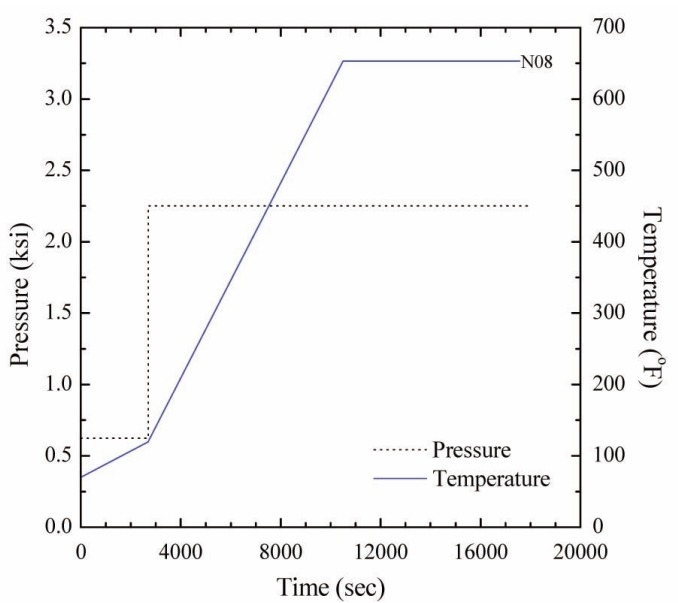

Fig. 2. Pressure and Temperature Histories of the Heat-up Process

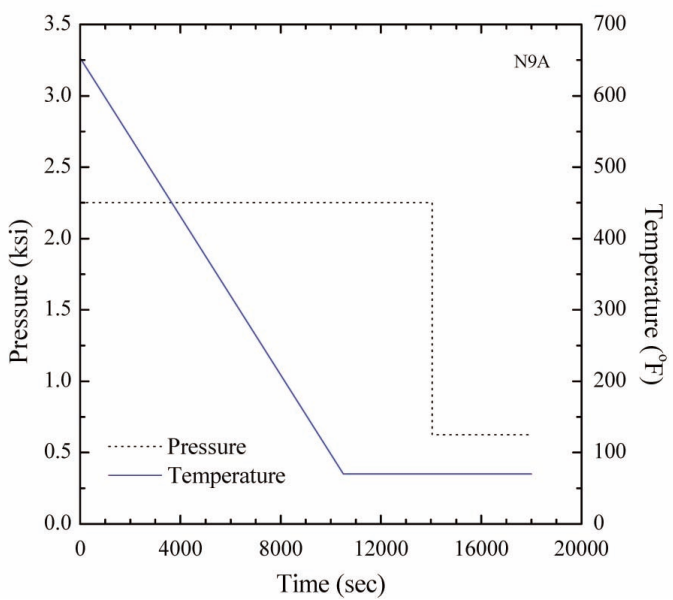

Fig. 3. Pressure and Temperature Histories of the Cool-down Process 
The heat transfer coefficient for natural convection on a vertical surface can be expressed as follows [3]:

$$
N u_{D}=\frac{h \cdot D}{k}
$$

Here, $N u_{D}, h, D$ and $k$ are the Nusselt number, heat transfer coefficient, characteristic dimension of the structure (i.e., diameter for a horizontal cylinder) and thermal conductivity, respectively. The average natural convection heat transfer coefficient can be represented in the following functional form for a variety of circumstances:

$$
N u_{D}=C \cdot(R a)^{n}
$$

In this equation, the values of the constants $C$ and $n$ are specified for each case, and $R a$ is the Rayleigh number defined as the product of the Grashof and Prandtl numbers:

$$
R a=G r \cdot P r
$$

The Grashof and Prandtl numbers are defined as

$$
\begin{gathered}
G r=\frac{D^{3} \cdot \rho^{2} \cdot g \cdot \beta \cdot \Delta T}{\mu^{2}}, \\
\operatorname{Pr}=\frac{c_{p} \cdot \mu}{k},
\end{gathered}
$$

where $\beta$ and $c_{p}$ are the isobaric cubic expansion coefficient and the specific heat, respectively [4].

Therefore, the heat transfer coefficient can be obtained from the relationships between Equations (1) through (5):

$$
h=\frac{C \cdot k}{D}\left(\frac{\rho^{2} \cdot g \cdot \beta}{\mu^{2}} \cdot \operatorname{Pr} \cdot \Delta T\right)^{n}
$$

Constants for use with Equation (6) for isothermal surfaces are $C=0.590, n=1 / 4$ for $10^{4}<\mathrm{Ra}<10^{9}$ and $C=0.100, n=$ $1 / 3$ for $10^{9}<R a<10^{13}$.

\subsection{Stress Analysis}

Using the temperature distributions determined during the thermal analysis, the stress analysis is performed with the inclusion of the pressure loads. Stresses are not obtained for all time steps used in the thermal analysis. Instead, time-point samplings are done according to ASME Section III, Subsection NB-3653.2 [5] to determine the most severe times, as follows:

For every pair of load sets, the peak stress intensity range $S_{p}$ is calculated as

$$
\begin{aligned}
S_{p}= & K_{1} C_{1} \frac{P_{o} D_{o}}{2 t}+K_{2} C_{2} \frac{D_{o}}{I} M_{i} \\
& +\frac{1}{2(1-v)} K_{3} E \alpha\left|\Delta T_{1}\right|, \\
& +K_{3} C_{3} E_{a b} \times\left|\alpha_{a} T_{a}-\alpha_{b} T_{b}\right| \\
& +\frac{1}{1-v} E \alpha\left|\Delta T_{2}\right|
\end{aligned}
$$

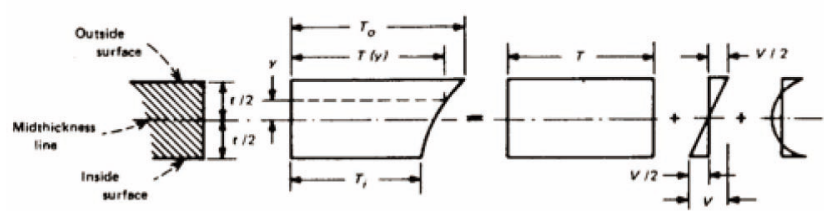

Fig. 4. Decomposition of the Temperature Distribution Range [5]

where $E$ is the modulus of elasticity; $\alpha$ is the mean coefficient of thermal expansion; $K_{1}, K_{2}$, and $K_{3}$ are local stress indices for the specific component under investigation; and $\Delta T_{1}$ and $\Delta T_{2}$ are determined by means of the process shown below. The temperature distribution range $T(y)$ can be thought of as being composed of three parts, as shown in Figure 4:

(1) A constant value,

$$
T=\frac{1}{t} \int_{-t / 2}^{t / 2} T(y) d y,
$$

which is the average value through the thickness. $T$ can be used in determining free thermal expansions., The values of $T$ thus determined (for the same pair of load sets) or two locations $\mathrm{a}$ and $\mathrm{b}$ on either side of a gross discontinuity can also be used for $T_{a}$ and $T_{b}$.

(2) A linear portion, with a zero average value, with the variation given by

$$
V=\frac{12}{t^{2}} \int_{-t / 2}^{t / 2} y T(y) d y
$$

(3) A nonlinear portion with a zero average value and a zero first moment with respect to the mid-thickness.

This decomposition of $T(y)$ into three parts is illustrated in Figure 4. The value of $\Delta T_{1}$ to be used in Equation (7) is the variation $V$ of the linear portion:

$$
\Delta T_{1}=V
$$

The value of $\Delta T_{2}$ to be used in Equation 7 is as follows:

$$
\begin{array}{r}
\Delta T_{2}=\max .\left(\left|T_{o}-T\right|-\frac{1}{2}\left|\Delta T_{1}\right|,\right. \\
\left.\left|T_{i}-T\right|-\frac{1}{2}\left|\Delta T_{1}\right|, 0\right)
\end{array}
$$

The alternating stress intensity $S_{a l t}$ is equal to one-half the value of $S_{p}$ calculated in Equation 7.

Several cases were chosen for the stress analysis. Therefore, a result file containing several cases is generated and is used for the ensuing fatigue analysis. A contour plot of the stress intensities for a typical transient case is shown in Figure 5.

\section{FATIGUE ASSESSMENT}

Cycle counting is used to summarize (an often lengthy 


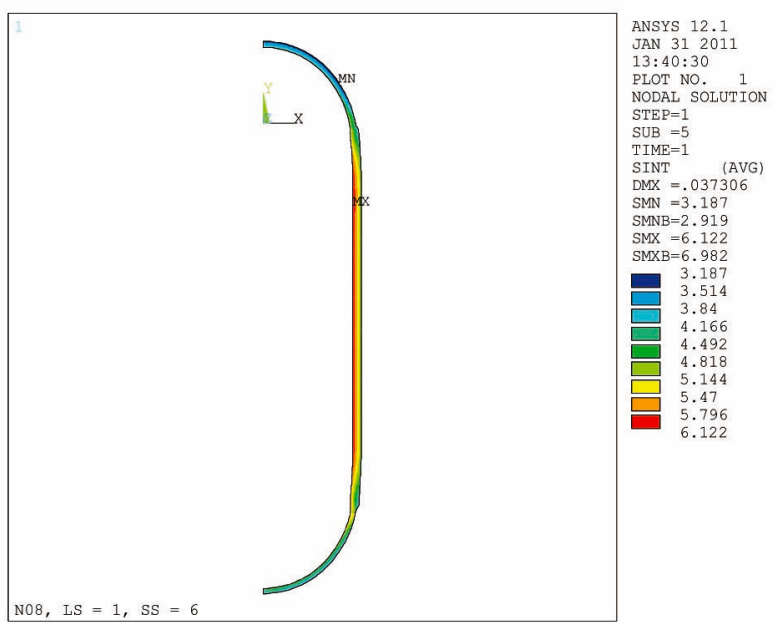

Fig. 5. Stress Contour of Load Step 1 for N08

process) irregular load-versus-time histories by providing the number of times cycles of various sizes that occur. The rain-flow method is used in this study [6]. The first step in implementing this procedure is to draw the stresstime history so that the time axis is oriented horizontally, with time increasing rightward. One can now imagine that the stress history forms a number of "pagoda roofs". Cycles are then defined by the manner in which rain is allowed to "drip" or "fall" down the roofs. A number of rules are imposed on the dripping rain so as to identify closed hysteresis loops. The rules specifying the manner in which rain falls are as follows:

(1) To eliminate the counting of half cycles, the stresstime history is drawn so as to begin and end at the stress value of the greatest magnitude.

(2) A flow of rain is begun at each stress reversal in the history and is allowed to continue to flow unless:

a. The rain began at a local maximum point (peak) and falls opposite to a local maximum point greater than that from which it came;

b. The rain began at a local minimum point (valley) and falls opposite to a local minimum point greater (in magnitude) than that from which it came;

c. It encounters a previous rain flow.

The foregoing procedure can be clarified through the use of an example. Figure 6 shows a stress history and the resulting flow of rain, where the given stress-time history begins and ends at the stress value of the greatest magnitude (point $A$ ). The rain flow is now initiated at each reversal in the stress history. Details of the cycle counting are summarized in Table 2.

Seven cuts are considered, as shown in Figure 1. They are chosen from experience for the locations with a structural discontinuity.

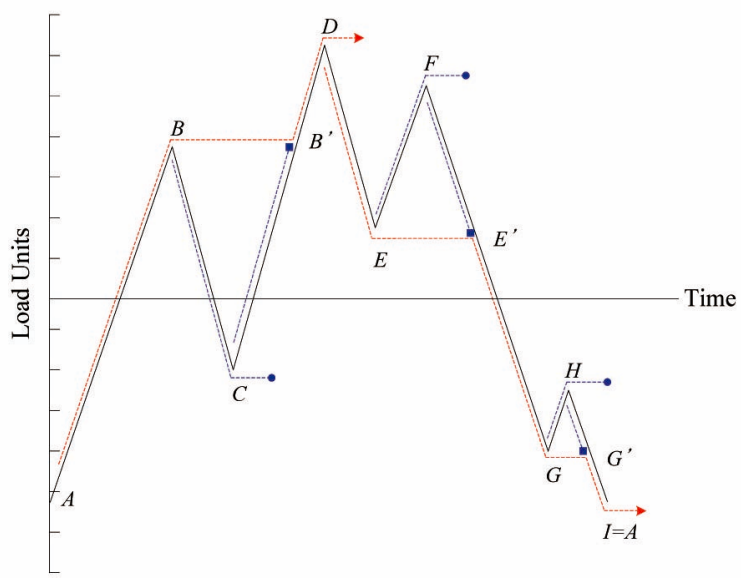

Fig. 6. Rain-flow Counting Example

Table 2. Summary of Cycle Countings

\begin{tabular}{c|c|c}
\hline Range (units) & Cycle Counts & Events \\
\hline 8 & 0.5 & $H-G$ \\
\hline 7 & 0.5 & $G-H$ \\
\hline 6 & 0.5 & $F-E$ \\
\hline 5 & 0.5 & $E-F$ \\
\hline 4 & 0.5 & $C-B$ \\
\hline 3 & 0.5 & $B-C$ \\
\hline 2 & 0.5 & $D-I$ \\
\hline 1 & 0.5 & $A-D$ \\
\hline
\end{tabular}

The stress concentration factor $K_{\beta}$ for the inclined shoulder at an angle $\beta$ to the normal direction is [7]

$$
K_{\beta}=1+\left(K_{0}-1\right)\left[1-\left(\frac{\beta}{90}\right)^{1+2.4 \sqrt{R / h}}\right],
$$

where $K_{0}$ is defined as

$$
K_{0}=1+\left[\frac{D / d-1}{2(2.8 D / d-2)} \cdot \frac{d}{R}\right]^{0.65}
$$

with $d, D, h, R$ and $\beta$ as shown in Figure 7 .

The stress concentration factors of Cuts B and F outside are calculated and are used in the fatigue evaluation.

The S-N curve, a curve of the alternating stress intensity $S_{a l t}=S_{p} / 2$ versus the allowable number of cycles is used, as shown in Figure 8 [5]. It is adjusted considering the design temperature.

The ranges of the primary plus the secondary stress 


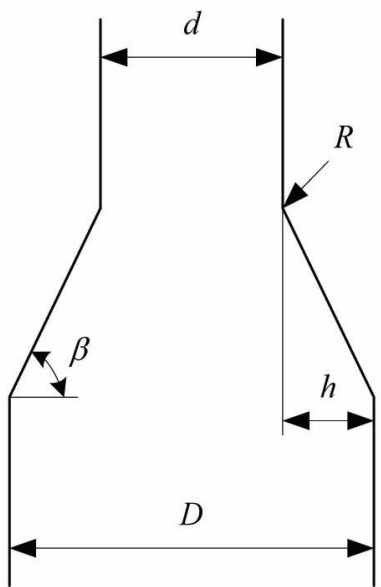

Fig. 7. Inclined Shoulder

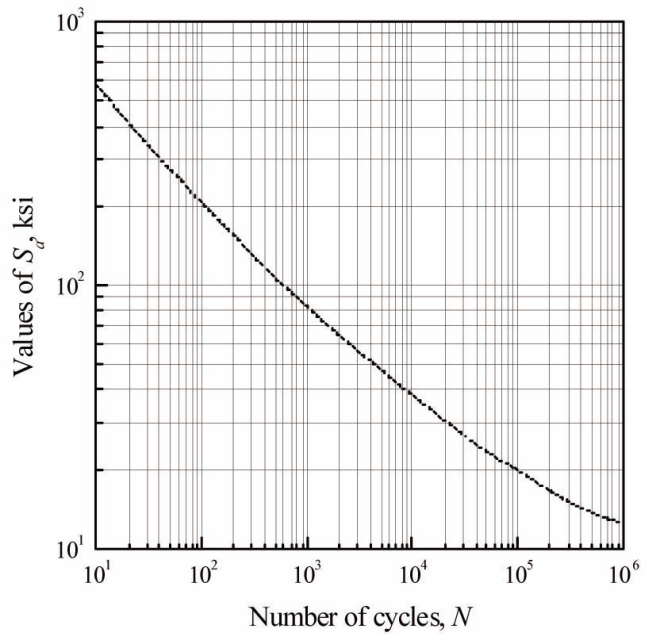

Fig. 8. Design Fatigue Curve

intensities are summarized in Figure 9, which shows that the stress levels in all locations investigated in this study are well below the allowable satisfying criteria.

When the stress concentration factors are not considered, the cumulative usage factors outside of Cut-B and Cut-F are 0.00699 and 0.02676 , respectively, which are very small compared to those of the case considered here. Therefore, the stress concentration factor is noted to be one of the most important factors affecting the fatigue usage factor.

Fatigue evaluations are performed when the thermal stress and the pressure stress are considered separately. In addition, the ranges of the primary plus the secondary stress intensities and the cumulative usage factors are investigated.

The cumulative usage factors are well below the allowable level of 1.0, as shown in Figure 10, satisfying the requirement. The comparison of the cumulative usage

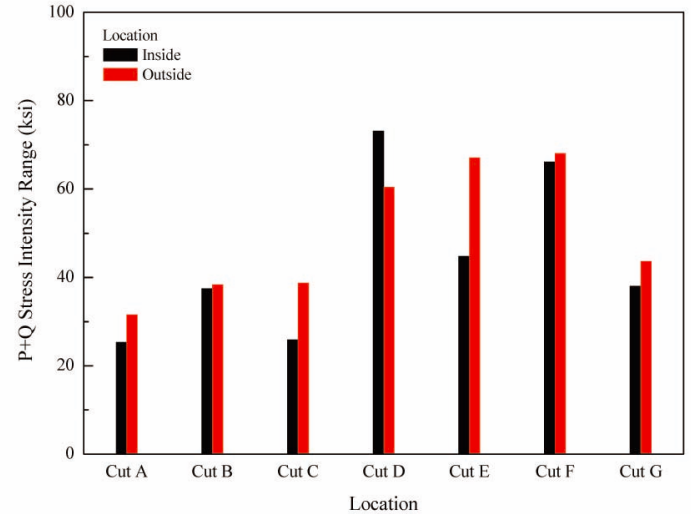

Fig. 9. Primary Plus Secondary Stress Intensities

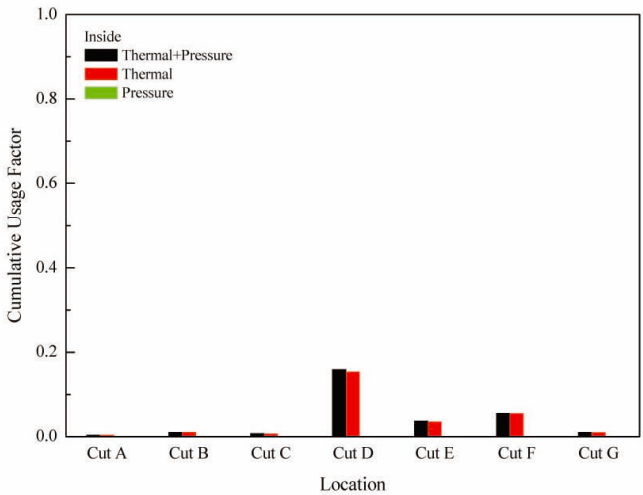

(a) Inner surface

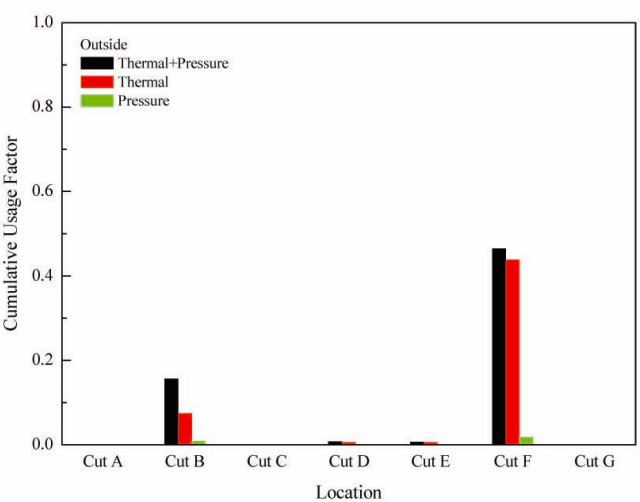

(b) Outer surface

Fig. 10. Comparison of the Cumulative usage Factors

factor among the pressure plus the thermal loadings, the thermal loading by itself, and pressure loading by itself are shown in Figure 10. Inside of the vessel, the pressure load does not affect the fatigue usage factor at all, whereas a slight pressure effect is observable outside. It was found that the majority of the fatigue usage factor comes from temperature variations during a transient. 


\section{CONCLUSIONS}

For a transient thermal analysis, the heat transfer coefficients are determined based on the operating environments and the thermal transient data are simplified to prepare a simple input deck. The most severe instances are found considering the satisfaction of total stress intensity range, and the stress levels at those times are obtained along with the applied pressure. These values are then used in the fatigue analysis to determine the final cumulative usage factor.

Example analyses of the reactor pressure vessel are performed for postulated arbitrary transients and two criteria are investigated, generating the following conclusions:

- The ranges of the primary plus the secondary stress intensities and the cumulative usage factors are well below the allowables.

- The major contribution to the fatigue usage factor is temperature variations during transients.

- No effect of pressure loading on the fatigue factor arises inside of the vessel.

- The stress concentration factor is one of the most important factors affecting the fatigue usage factor.

\section{REFERENCES}

[1] IAEA, Status Report for Advanced Nuclear Reactor Designs - Report 77 - System-Integrated Modular Advanced Reactor (SMART), http://aris.iaea.org/ ARIS/, 2011, International Atomic Energy Agency.

[2] ANSYS, Inc., Theory Reference for ANSYS and ANSYS Workbench Release 12.0, 2009, Canonsburg, PA.

[ 3 ] Holman, J.P., Heat Transfer, $9^{\text {th }}$ ed., 2002, McGrawHill, London.

[4] Wagner, W., Kretzschmar, H-J., International Steam Tables - Properties of Water and Steam based on the Industrial Formulation IAPWS-IF97, $2^{\text {nd }}$ ed., 2008, Springer.

[5] ASME, ASME Boiler and Pressure Vessel Code, Section III Rules for Construction of Nuclear Power Plant Components, Division 1, Subsection NB Class 1 Components, 2004, The American Society of Mechanical Engineers.

[6] Bannantine, J.A., Comer, J.J., Handrock, J.L., Fundamentals of Metal Fatigue Analysis, 1989, Prentice Hall, New Jersey.

[ 7 ] Heywood, R.B., Designing by Photoelasticity, 1952, Chapman \& Hall Ltd., London. 